\title{
Constructive Empiricism, Partial Structures and the Modal Interpretation of Quantum Mechanics
}

\author{
Otávio Bueno
}

Department of Philosophy, University of Miami, Coral Gables, FL 33124, USA. E-mail: otaviobueno@me.com

Editors: Eliahu Cohen \& Danko Georgiev

Article history: Submitted on October 10, 2013; Accepted on December 13, 2013; Published on January 15, 2014.

$\mathrm{V}$ an Fraassen's modal interpretation of nonrelativistic quantum mechanics is articulated to support an anti-realist account of quantum theory. However, given the particular form of van Fraassen's anti-realism (constructive empiricism), two problems arise when we try to make it compatible with the modal interpretation: one difficulty concerns the tension between the need for modal operators in the modal interpretation and van Fraassen's skepticism regarding real modality in nature; another addresses the need for the truth predicate in the modal interpretation and van Fraassen's rejection of truth as the aim of science. After examining these two problems, I suggest a formal framework in which they can be accommodated-using da Costa and French's partial structures approach-and indicate a variant of van Fraassen's modal interpretation that does not face these difficulties.

Quanta 2014; 3: 1-15.

\section{Introduction}

The quantum-logical approach to the foundations of nonrelativistic quantum mechanics has provided rich insights into our understanding of quantum theory. (Here, I will only consider non-relativistic quantum mechanics. Reference to quantum mechanics is restricted to this class of theories and their interpretations.) The quantum-logical approach has indicated mathematical structures that are often used in the formulation of quantum mechanics (such as orthomodular lattices), and clarified the relationship between these structures and logic [1-5]. Just as in classical mechanics the algebra of states of a physical system is a Boolean algebra, in quantum mechanics, the corresponding algebra is a non-distributive, orthomodular lattice. The corresponding logic-the consequence relation defined for the theory's language-is then not classical, but quantum. (It is a delicate issue to determine the language of quantum mechanics. According to certain authors, there is no such thing. The language of the theory is only a fragment of functional analysis [6, pp.83-85]. This is part and parcel of the peculiarities of quantum mechanics. For example, given that quantum particles lack identity conditions (at least in one interpretation of the theory), the language of quantum mechanics cannot be classical, but it has to accommodate objects for which identity does not meaningfully apply [7,-11].)

The motivation for the shift in logic is the same underlying the construction of other interpretations of quantum

(c) This is an open access article distributed under the terms of the Creative Commons Attribution License CC-BY-3.0, which permits unrestricted use, distribution, and reproduction in any medium, provided the original author and source are credited. 
mechanics, namely, to solve conceptual difficulties facing the theory; in particular, (1) to provide an account of measurement, (2) to accommodate the well-known paradoxes (such as EPR and Schrödinger's), and (3) to examine the issue of identity and non-individuality of quantum particles. It has to be admitted that, from the quantum-logical point of view, these issues have not been as thoroughly examined as they could (most work concentrates on the issue of measurement), and that exaggerated claims have been made on the basis of quantum logic (for a critical appraisal, see [12,13]). However, overall, the approach still provides important insights, since it suggests a unified picture to examine the foundations of quantum mechanics.

Independently of the merits of the quantum-logical approach, it is important to distinguish quantum logic as a particular interpretation of quantum mechanics from quantum logic as a methodological tool to investigate the theory. As an interpretation, Putnam [3] argued that quantum logic provides an argument to support realism in quantum mechanics. Roughly speaking, this is because in this approach all observables can be assumed to have sharp values. (Putnam's move towards realism is correctly criticised in [14]; see also [12].) As a methodological tool, we can use the structures and techniques provided by quantum logic as conceptual resources to explore the quantum domain, without assuming the interpretative moves typically made by quantum logicians. Admittedly, the distinction is somewhat vague, since the interpretative claims from quantum logic are based on the use of (quantum) logical tools. However, as often happens, the (logical) formalism does not single out one unique (quantum-logical) interpretation, but underdetermines several possible interpretations. Thus, using the methodological tools of quantum logic, we are able to provide alternative interpretations of quantum mechanicsresisting, for instance, Putnam's move towards realism.

In this paper, I want to explore one aspect of quantum logic: its role in the formulation of the modal interpretation of quantum mechanics. Instead of assuming the interpretative claims of the quantum-logical approach, I shall use it as a method to construct one kind of interpretation. Although the modal interpretation is often associated with realism in quantum mechanics [15], it was first formulated by van Fraassen as an anti-realist proposal [13, 16-18]. And it is in the anti-realist camp that it belongs more comfortably. However, as we shall see, given the particular form of van Fraassen's constructive empiricism [19, 20], two problems arise: one difficulty concerns the tension between the need for modal operators in the modal interpretation and van Fraassen's skepticism with regard to real modality in nature [20]; another addresses the need for the truth predicate in the modal interpretation and van Fraassen's rejection of truth as the aim of science [19]. After examining these two problems, I will suggest a formal framework in which they can be accommodated-using da Costa and French's partial structures and quasi-truth [21-23]-and which provides a variant of van Fraassen's modal interpretation. Given the role played by structures in this new account, I shall call it a modal-structural interpretation.

\section{Quantum Logic, Empiricism and the Modal Interpretation of Quantum Mechanics}

In order to develop a new formulation of empiricism, van Fraassen articulated a proposal to overcome the major shortcoming of previous empiricist accounts: to accommodate the theoretical aspects of science within the bounds of empiricism. The difficulty is that theoretical talk cannot be eliminated from the scientific description of the world without radically depriving science from its actual content, nor can it be reduced to purely empirical factors. The early attempts, in the hands of logical positivists, to reduce the theoretical content of science to observation reports failed for well-known reasons (ultimately, science cannot be regimented in the restricted framework assumed by positivism). The alternative suggested by van Fraassen consists in broadening the limits of empiricism, and rejecting the reductionism of former approaches.

There are two central features of van Fraassen's approach that I wish to emphasize. First, as is well known, instead of trying to reduce the theoretical aspects of science to empirical phenomena, van Fraassen's strategy is to change the aim of science, but still stressing the role of observation to achieve this aim. According to his proposal (constructive empiricism), the aim of science is not truth, but something weaker: empirical adequacy [19, p.12]. Using the resources of the semantic approach (according to which to present a theory is to specify a family of structures, its models [19, p.64]), van Fraassen characterizes this concept in the following way. A theory is empirically adequate if it has a model such that its empirical substructures are isomorphic to the structures that represent empirical phenomena ( [19, p.64]; for a discussion, see [24]). In this way, the empirical adequacy of a theory reflects one role that empirical information plays in science: to provide constraints for theory acceptance. Now, empirical adequacy is strictly weaker than truth, and it is reducible to truth only if we are dealing with observable phenomena [19, p.72]. Thus, the search for empirically adequate theories does not entail the commitment to unobservable entities. For suppose that there 
are no unobservable entities in the world; a false theory, which postulates the existence of these entities, can still be empirically adequate. In this sense, by taking empirical adequacy as the aim of science, van Fraassen is able to provide a proposal in which empirical factors have a crucial role to play in science without collapsing into a reduction of theory to observation.

The avoidance of reductionism allows van Fraassen to put forward a crucial argument against realism: the underdetermination argument. Because there is a gap between certain theories (such as classical mechanics or non-relativistic quantum mechanics and their interpretations) and empirical phenomena, empirically equivalent but conceptually distinct theories may arise. And the empiricist sees no epistemic reason to select one of them; after all, as far as the empirical phenomena are concerned, they are equally adequate [19]. (Note that van Fraassen only uses the argument from underdetermination in very specific contexts, invoking particular theories to make his case. The argument is never used as a feature of every theory, applied to science as a whole [25, pp.346-347].) A great deal can be said, and has been said, about this argument, but for our present purposes it is enough to indicate the role it plays in constructive empiricism. The gap between theoretical models and models of the phenomena allows the empiricist to remain agnostic about the existence of unobservable entities, which are postulated in some descriptions of the phenomena, but not in other, also empirically adequate, descriptions.

This leads to the second empiricist feature of van Fraassen's approach that I wish to examine. Although the constructive empiricist is only agnostic about the existence of unobservable entities (as van Fraassen points out: "I wish merely to be agnostic about the existence of the unobservable aspects of the world described by science" [19, p.72]), he is entirely skeptical about the existence of real modality in nature, such as laws of nature and objective chance. In van Fraassen's own words:

To be an empiricist is to withhold belief in anything that goes beyond the actual, observable phenomena, and to recognize no objective modality in nature. ( [19, pp.202-203]; the italics are mine; see also [20]. A formulation of empiricism in terms of a stance rather than a doctrine to be believed is developed in [26].)

To recognize real modality in nature is to recognize the existence of non-actual, merely possible phenomena, and also to recognize necessary connections in reality. These are, of course, anathema to empiricism ever since Hume. Thus van Fraassen rehearses here a characteristic point of the empiricist tradition, although with a new twist. Among the real modality the empiricist is critical of, laws of nature are prominent, and with the emergence of quantum mechanics, a new alternative could be provided. After all, given the crucial role played by symmetry in the construction of quantum mechanics, van Fraassen has grounds to propose, roughly speaking, that the concept of a law of nature could be replaced, in some contexts, by the concept of symmetry [20]. Objective modality, such as laws, are not only philosophically problematic [20, pp.16-128], but can also be replaced, in some contexts, by a metaphysically less problematic alternative, based on symmetry considerations [20, pp.216-289].

Now, because quantum mechanics provides a crucial case for van Fraassen's approach, and because of its overall importance in science, it is understandable that the empiricist be expected to provide an interpretation of it. In this context, van Fraassen formulates and develops the modal interpretation of quantum mechanics. The interpretation is presented as an implementation of central features of van Fraassen's general empiricist approach, and I now discuss some of its distinctive traits. (In the preface to [20], van Fraassen notes: "This book was originally twice as long. When a general approach is announced and advocated, it remains hand-waving except to the extent that it is implemented. Accordingly, the now missing part was devoted to a detailed study of the structure and interpretation of quantum mechanics. It will appear separately, as Quantum Mechanics: An Empiricist View [20, pp.vii-viii].)

The modal interpretation is explicitly articulated as an alternative to von Neumann's interpretation rule [1]. This rule provides a close link between observables and states of a physical system, indicating how to read assignments of values to observables (essentially, value assignments classify the states). The link is as follows:

Observable $B$ has value $b$ iff a measurement of $B$ is certain to have outcome $b$. (For a discussion, see [13, pp.274-278].)

The crucial feature, as van Fraassen notes, is the logical form of this interpretation rule: a biconditional linking assignments of values to observables and outcomes of measurements. The (apparent) classical flavor of this link is noticeable. However, what happens if a measurement is not certain to have a given outcome? To this question, von Neumann provides a peculiar answer: in this case, the observable has no value. The classical flavor of the interpretation is therefore deceptive: not all observables have a value after all-unmeasured observables have no value, excluding the case of certainty [13, p.274].

The main feature of the modal interpretation is then to distinguish between two concepts of state: value and dynamic states. The distinction is motivated by the fact that, in the case of quantum mechanics, (1) we cannot 
assume that observables have values, 'there to be seen if we look', nor can we (2) assume that the evolution of the physical system is determined completely by what those values are. These assumptions may have been taken for granted in the case of classical mechanics, but the picture changes once we move to the quantum domain. To each of these assumptions, there corresponds a particular concept of state. The value state is fully specified by stating which observables have values, and which they are; the dynamic state is completely determined by stating how the system will develop if acted upon in a particular way, and how it will develop if isolated [13, p.275].

Corresponding to these two concepts of state, we have two types of proposition: a value-attributing proposition, denoted by $\langle m, E\rangle$, which states that observable $m$ actually has a value in $E$ (where $E$ is, typically, a Borel set); and a state-attributing proposition, denoted by $[m, E]$, according to which the state is such that a measurement of $m$ must have a value in $E$ [13, p.275]. These propositions have a well-determined body of truth-makers: valueattributing propositions are true (or false) depending on value states, whereas the truth-values of state-attributions depend on dynamic states.

If von Neumann's interpretation rule is rejected, the equivalence between value-attributing and stateattributing propositions is denied. Only one side of the biconditional holds. But what side? Since measurement outcomes are relevant to what state the system is in, it is natural that $[m, E]$ implies $\langle m, E\rangle$; that is, if the state is such that a measurement of $m$ must have a value in $E$, then $m$ does have a value in $E$ [13, p.276]. In other words, in von Neumann's interpretation rule only the right-to-left conditional holds. This allows van Fraassen to introduce unsharp values to observables. After all, if $[m, E]$ is not true (that is, if the state is not such that $m$ must have a value in $E$ ), it is still possible that $m$ does have a value in $E$, although this value may be unsharp [13, pp.276;282283]. Furthermore, crucial information about the physical system is provided by the dynamic state, which still remains the basic state to consider, since it gives information about the system's evolution. In this sense, the importance of the actual values of observables derives from the fact that they provide indications about the dynamic state. And once we know that state, we are in a position to know how the system will evolve (either in isolation or in interaction with another system).

Far more can be said, of course, about the modal interpretation; in particular, how it handles the measurement problem [13, pp.283-299;317-337], the EPR paradox [13, pp.338-374], and the issues of identity and nonindividuality of quantum particles [13, pp.375-482]. But instead of going into these issues here, I will consider another pressing point: what are the empiricist features in the modal interpretation? Two of them deserve notice. The first derives from van Fraassen's examination of the empirical basis of quantum theory [13, §4-5]. As he argues, there are possible phenomena in the domain of quantum mechanics that cannot be accommodated by any causal model (in a minimal sense of causality). (These are common cause models, which spell out at least necessary conditions for causality. A correlation between events $A$ and $B$ has a common cause in factor $C$ if: (1) $C$ precedes $A$ and $B$; (2) $P(A \mid C)>P(A \mid \neg C)$ and $P(B \mid C)>P(B \mid \neg C)$; and (3) $P(A \wedge B \mid C)=P(A \mid C) P(B \mid C)$, where $P(X \mid Y)$ is the conditional probability of $X$ given $Y[13$, pp.53-57;8185]. If it is argued that common causes do not provide even a necessary condition for causality, it can be replied that the notion of causality under consideration is then decidedly too weak [13, p.487, note 4].) Due to empirical considerations, theories articulated in terms of common cause models are shown to be inadequate, in the sense that the structures describing the data of quantum phenomena cannot be embedded into the theoretical models.

The second empiricist feature comes in with the introduction of modality. As an interpretation of quantum theory, the modal interpretation spells out how the world could be if quantum mechanics were true [13, p.242]. It explores possibilities in the way the theory pictures the world (for instance, in what respect quantum theory is indeterministic, in what respect it is not), extending the account provided by the theory to unobservable factors, in such a way that a fuller picture of the world is presented. In this sense, the modal interpretation increases our understanding of quantum mechanics. Roughly speaking, the empiricist component enters with the claim that, with regard to unobservable entities (such as elementary particles), it is enough to say how they can be. (The epigraph of [13] is precisely this statement of Descartes's: "Que, touchant les choses que nos sens n'aperçoivent point, il suffit d'expliquer comment elles peuvent être" (Principles, iv, 204; "That, concerning those things that our senses do not perceive, it is enough to explain how they can be"). This is, of course, a thoroughly empiricist point.) In this way, a commitment to the existence of these entities is not required, since the theory can be interpreted so that what it states about the phenomena are only certain possibilities (probabilities of measurement outcomes, relative possibilities of trajectories of a given system etc.).

However, despite these empiricist features in van Fraassen's interpretation, I think the modal interpretation faces two problems if we try to make it compatible with constructive empiricism. To these issues I should turn now. 


\section{Two Problems: Truth and Modality}

The first problem is concerned with the notion of truth, and its role in the modal interpretation. The second concerns modality: its status and the way it is introduced into van Fraassen's proposal. I argue that there is a tension between these two moves, and suggest a way out using the partial structures framework. It goes without saying that van Fraassen has provided a strong case for understanding quantum mechanics in terms of certain modal operators, and his search for an empiricist view of the theory in a decidedly classical setting (with regard to logic and probability) is significant. (As van Fraassen argues, quantum theory itself does not require us to abandon either classical logic or classical probability theory [13, pp.134135].) The point is not uncontentious, but as he points out, quantum theory is compatible with the adoption of a non-classical logic (such as quantum logic) as a fragment of a broader logic that is classical.) None of this is in question here. My point is to suggest an alternative empiricist formulation of the modal interpretation, which preserves as much as possible of van Fraassen's positive program, without being subject to the philosophical difficulties found in it.

\subsection{Truth}

Before spelling out these difficulties, note an important feature of the distinction between state attributions and value attributions of a physical system. First, state attributions are theoretical constructs, and part of the worries involved in theory construction arises from the proper representation of these states. The point here is that, in order to accommodate the claim that a given physical system is in a certain state (state attribution), and to distinguish this statement from the system having a certain value (value attribution), the notion of truth is introduced. As we saw, the connection between a value state and a value-attributing proposition is that value states are truthmakers of value-attributing propositions [13, pp.275-276]. Similarly, dynamic states and state-attributing propositions are connected by the fact that the former are what make the latter true.

In other words, the value-state distinction is cashed out in terms of truth. As van Fraassen points out [13, pp.280281], if a physical system $X$ has dynamic state (represented by a density operator) $W$ at a time $t$, the stateattributions $[M, E]$ which are true are those such that $\operatorname{Tr}\left(W I_{E}^{M}\right)=1$. (A few comments about the notation: (a) $\mathrm{Tr}$ is a linear map, called trace, of operators into numbers, which give us the probability that a measurement of $m$ has a value in $E$. (b) $I$ is a projection operator, which is a Her- mitian operator $I$ such that $I I=I$. (c) $I_{E}^{M}$ is then defined as the Hermitian operator $I$ such that $I x$ is $x$ if $M x=a x$ for some value $a$ in $E$, and is the zero vector if $M x=b x$ for some value $b$ outside $E$, where $E$ is a Borel set. (d) That the trace function $\operatorname{Tr}$ provides a probability is due to the following equations: $P_{x}^{m}(E)=\left(x \cdot I_{E}^{M} x\right)=\operatorname{Tr}\left(I_{x}^{M} I_{E}^{M}\right)$, where $P_{x}^{m}(E)$ is the probability that a measurement of $m$ has a value in $E ;\left(x \cdot I_{E}^{M} x\right)$ is the inner product of $x$ and $I_{E}^{M} x$; and $I_{x}^{M}$ is the projection on the subspace $[x]$ spanned by $x$. For details, see [13, pp.147-152;157-165;280-281].) As opposed to state-attributions, value-attributions cannot be deduced from the dynamic state. But, according to van Fraassen, they are constrained in three ways, which again are spelled out in terms of truth: (1) If $[M, E]$ is true, so is the value-attribution $\langle M, E\rangle$, that is, observable $M$ has value in $E$; (2) all true value-attributions could have probability 1 together; and (3) the set of true value-attributions is maximal with respect to feature (2) [13, p.281]. So, the assignment of truth-conditions to state-attributing and value-attributing propositions is crucial to spell out the difference between them (the former, but not the latter, can be deduced from the dynamic state).

But how can we make sense, in empiricist terms, of these truth-conditions? As we saw, for the constructive empiricist, science does not aim at the truth, but at empirical adequacy. In van Fraassen's formulation, a theory $T$ is empirically adequate if there is a model of $T$ such that all the phenomena (properly structured) are isomorphic to the empirical substructures of this model [19, p.64]. The idea is that empirical adequacy, as defined, is reduced to truth if we consider only observable phenomena [13, p.4]. Note, however, that in van Fraassen's formulation, empirical adequacy is a model-theoretic notion, and therefore we are dealing here with the notion of truth in a structure. Thus, empirical adequacy can be reduced at best to this notion of truth, and not to truth simpliciter. It may be argued that we can define the latter in terms of the former by stating that a sentence $\alpha$ is true simpliciter if it is true in the structure @ of the actual world (supposing that there is one). The problem is that an empiricist cannot accept this formulation without a severe qualification, since presumably the structure @ incorporates information about unobservables which the empiricist is agnostic about. So the distinction between observable and unobservable entities would have to be drawn with regard to @, and we would be back to the properties of the structure in question, and hence to the notion of truth in a structure. I conclude that if empirical adequacy is reducible to truth in van Fraassen's account, the latter cannot be truth simpliciter.

Now I raise this point because, as van Fraassen acknowledges, state attributions are theoretical constructs, and arguably involve reference to unobservables (in fact, 
a state is represented by a statistical operator, which is typically a particular kind of Hermitian operator defined on a Hilbert space). However, it is not clear how we should understand, in empiricist terms, the claim that state attributions are true. In order not to be committed to the existence of unobservables involved in such attributions, the empiricist would have to distinguish, in the structure which describes a state attribution, an empirical substructure from a theoretical superstructure. But it is clear that this cannot be done, since state attributions, as such, are entirely theoretical constructions, and they do not seem to incorporate an empirical part. The point here is that, in order not to be committed to the theoretical content involved in state attributions, the empiricist should avoid talking about their truth, and should run the semantic analysis in terms of a weaker notion. My suggestion, to be spelled out below, is to change here the norm of theoretical talk to quasi-truth, rather than truth. (Someone may argue that van Fraassen does not need a substantial notion of truth to assign truth-values to stateattributing and value-attributing propositions. He can simply adopt a disquotational approach. The suggestion is, of course, well motivated, given that, in some writings of van Fraassen, the disquotational account plays some role [27, 28]. But the suggestion faces a serious problem. As we have just seen, in order for the notion of empirical adequacy to be reducible to truth (when we only consider observable phenomena), the empiricist cannot adopt the notion of truth simpliciter. After all, as cashed out by van Fraassen, empirical adequacy is a model-theoretic notion, and so truth has to be understood in a model-theoretic way. Given that a disquotational account is not modeltheoretic, it will not help the empiricist here. For what is needed for an interpretation of state and value attributions is the notion of truth in a structure. However, as argued above, this notion is too strong for the empiricist to use when talking about unobservable entities.)

\subsection{Modality}

Before discussing the concept of quasi-truth, let me consider the second feature of the modal interpretation: the introduction of modal operators. Since van Fraassen is skeptical about the existence of objective modality, all modal talk involved in his interpretation comes from language, as it were. As he points out, modal talk is talk about the structure of our own ways of representing the phenomena [20, pp.68;92;213-214;223]. It is not taken to correspond to something real, such as a possible world in Lewis's sense [29], nor is it taken to be primitive (else van Fraassen's position would lead to modalism). Indeed, countenancing possible worlds would be incompatible with a thoroughly empiricist outlook, given the increase in the ontological commitments that they bring. And admitting a primitive notion of modality would be equally problematic, since if this notion has any metaphysical import, it will clash with van Fraassen's skepticism about modality (in particular, with the rejection of the notion of objective chance).

It may be argued that constructive empiricism is compatible with Lewis's modal realism [30]. After all, strictly speaking, constructive empiricism is only a claim about the aim of science-involving the search for empirically adequate theories and restricting belief to the empirical adequacy of the relevant scientific theories [19, p.12]. Modal realism is compatible with that aim for science, just as a number of other metaphysical views are. Although this is literally correct, the point of constructive empiricism is to develop an empiricist account of science, and a significant feature of the empiricist stance is to be suspicious precisely of the kind of postulational metaphysics advanced by the modal realist [26]. As a result, it seems unmotivated and questionable for the constructive empiricist to embrace modal realism. A less metaphysically loaded approach seems in order.

It is not surprising that modal operators are introduced in van Fraassen's interpretation of quantum mechanics as algebraic devices; that is, constructions which mathematically have the form of modal operators in modal logic, but which lack any other (metaphysical) warrant. Van Fraassen introduces two kinds of modal operators, one of them based on a transitive relative possibility relation $R$ (between possible situations), and another based on an equivalence relation (namely, equality of dynamic states). Let me briefly spell this out.

The modal interpretation, formulated by van Fraassen in quantum-logical terms, has the notion of possible situation as basic [13, p.302]. A proposition is then identified with a set of situations; intuitively, those situations in which the proposition is true. We say that a proposition $q$ is true in a situation $w$ exactly if $w$ is in $q$ [13, p.302]. Let $\mathbf{V}$ be the set of value-attributions, i.e. $\mathbf{V}=\{\langle m, E\rangle$ : $m$ is an observable and $E$ is a Borel set $\}$, and $\mathbf{P}$ the set of state-attributions: $\mathrm{P}=\{[m, E]: m$ is an observable and $E$ is a Borel set\}. Now, according to the modal interpretation, a situation $w$ is possible relative to a situation $w^{\prime}$ (in symbols, $w R w^{\prime}$ ) if for all $q$ in $\mathbf{V}$, if $w$ is in $q$, then $w^{\prime}$ is in $q$ [13, p.311]. In terms of this notion, modal operators of necessity $\square$ and possibility $\diamond$ are then defined for all propositions [13, p.314]. Indeed, if $q$ is a proposition and $w$ a possible situation, we say that

$$
\begin{array}{r}
\square q=\left\{w: \text { for all } w^{\prime}, \text { if } w R w^{\prime}, \text { then } w^{\prime} \in q\right\} \\
\diamond q=\left\{w: \text { for some } w^{\prime}, \text { if } w R w^{\prime}, \text { then } w^{\prime} \in q\right\}
\end{array}
$$


This establishes at once a connection between stateattributing and value-attributing propositions, namely: $[m, E]=\square\langle m, E\rangle$ (a proof can be found in [13, p.316]). The second kind of modal operator introduced is based on an equivalence relation; namely, equality of a dynamic state $x$ with respect to situations $w$ and $w^{\prime}$. Indeed, if $q$ is a proposition, we say that $\square q=\{w$ : for all $w^{\prime}$, if $x(w)=x\left(w^{\prime}\right)$, then $\left.w^{\prime} \in q\right\}$. As a consequence, a similar result also holds for this necessity operator: $[m, E]=\square\langle m, E\rangle[13$, pp.316-317].

From this brief exposition, it should be clear that all the modal operators introduced in the modal interpretation are only algebraic constructs, called modal because of the formal similarities that they bear to the necessity and possibility operators in modal logic. This may well be all right given van Fraassen's skepticism about modality. After all, as noted, van Fraassen is not willing to be committed to the existence of a modal reality to which these operators refer. But is this algebraic similarity enough to characterize these operators as modal?

There is certainly a whole story to be told here, but perhaps it suffices to note a few points. We are, of course, allowed to call modal whatever operators satisfying certain formal conditions, and provided that we are only concerned with particular formal results, there is certainly no problem with this. But the issue arises as to how we should interpret these operators, and what consequences to draw about their status. Once we enter this debate, we are doing metaphysics-not logic or the foundations of physics. And at this level, as Lewis points out [29, pp.1720], we need more than purely formal considerations. (According to Lewis: "Where we need possible worlds $[\ldots]$ is in applying the results of [...] metalogical investigations. Metalogical results, by themselves, answer no questions about the logic of modality. They give us conditional answers only: if modal operators can be correctly analysed in so-and-so way, then they obey so-and-so system of modal logic. We must consider whether they may indeed be so analysed; and then we are doing metaphysics, not mathematics" [29, p.17]) Now, as noted, van Fraassen surely has an account of modality to offer [20,31]. What is not yet clear is how the modal operators introduced in his interpretation mesh with the idea that modality is a feature of our language (of the structures used to represent the phenomena), and do not refer to independently existing possible entities. For the necessity operator $\square$ was defined in terms of (a property of a) dynamic state, which is a theoretical construct and bears a clear relation not to any linguistic entity, but to a physical event. In this sense, we are apparently in a situation in which we are reducing modality to certain physical circumstances (or, at least, to events which depend on such circumstances). And this seems at odds with the idea that necessity is a lin- guistic feature. Moreover, the two other modal operators, $\square$ and $\diamond$, are explicitly defined in terms of a relation of relative possibility among situations. But, as we saw, the notion of possible situation is primitive, and it is not clear how it relates either to physical or to linguistic events. If it is argued that these situations function only as an index set, and hence have no metaphysical import, we are back to the initial position in which no interpretation of the modal operators was offered. If these situations are to have any explanatory role vis-à-vis the status of the modal operators, we need an account of them. A story should be told about their status-otherwise, we will end up with no properly modal notions. (It might be argued that in order to introduce modal operators into the modal interpretation, van Fraassen can use the idea that when we accept a probabilistic theory (such as quantum mechanics) we accept it as providing a panel of expert functions to guide our personal probability, our opinion [20]. The modal operators introduced can be interpreted in terms of the expert functions: they guide our personal probability as to the observable predictions of quantum theory. But there are two problems with this suggestion. First, it does not really allow us to interpret the modal operators, since the latter apply to value-attributing and state-attributing propositions, which describe unobservable properties of a quantum system. The empiricist twist in introducing expert functions is to restrict them to observable features of the theory, but that is what the modal operators violate. Second, if empiricists adopt expert functions as an account of modal operators, they will make quantum mechanics entirely subjective. For quantum mechanics would then be a theory about the change of our opinion with regard to quantum states, rather than a theory about quantum phenomena themselves.)

But why does van Fraassen need to introduce modal operators in his interpretation of quantum mechanics? There are several reasons for that. These operators allow him to stress the empiricist status of his interpretation of quantum mechanics, since they cash out the claim that at most quantum theory tells us how the quantum world can be. Of course, as van Fraassen notes, any interpretation provides an account of how the world can be if the theory is true [13]. What is special about the modal interpretation, in van Fraassen's formulation, is that this is the most that the theory provides. The empiricist sentiment expressed by Descartes in the epigraph of [13], which was quoted above, underscores the empiricist nature of van Fraassen's interpretation.

Furthermore, it is via modal notions that van Fraassen talks about observables having unsharp values. One could cash out the talk of unsharp values of observables in terms of partial functions. Van Fraassen acknowledges this option but does not adopt it [13, p.307], articulating 
instead an account that leads more straightforwardly to the introduction of probability in quantum mechanics. Given that probability is a modality-a possibility with degrees [19, §6]-it is ultimately for reasons having to do with the introduction of modality that unsharp values are cashed out the way they are.

\subsection{Truth and Modality: Theoretical Constraints}

Can constructive empiricists make sense of applying truth to unobservable entities, such as dynamic states? It may be argued that they can as long as none of these claims (that refer to unobservable objects) is ever asserted. What this amounts to is that, although claims referring to unobservable entities are truth-apt, they are not to be evaluated in terms of their truth or falsity: some other norm has to be introduced. Which norm? Clearly empirical adequacy will not do. As formulated by van Fraassen, empirical adequacy is a property of a (class of) model(s), not a property of a particular statement [19, p.64]. Now, even if we consider the models of a proposition about dynamic states, it is not obvious that we can determine an empirical substructure corresponding to this model. As van Fraassen notes, states can be identified in terms of observables, but not with observables [13]. So empirical adequacy does not seem to be an adequate norm to evaluate interpretative claims about quantum mechanics, at least from an empiricist point of view.

This raises the broader issue: how should one evaluate interpretations of quantum mechanics (according to the empiricist)? Clearly, the interpretations cannot be taken to be true, even if they are truth-apt. For they refer to unobservable entities, which empiricists are agnostic about. Can empiricists claim that interpretations are true, but they do not believe in them? Clearly, being a kind of Moore's paradox, this is not coherent. Can empiricists claim that an interpretation, although not true, is still useful (in providing us with understanding of the quantum world)? Certainly; but an account has to be provided as to how fictions (since interpretations are not true) can increase our understanding. For example, how do literary fictions increase our understanding? One needs a notion of truth, or some modal notion, to cash this out. But this is precisely the problem in the first place! Let us see why either the notion of truth or some modal notion is required here.

Let us consider an example. Suppose that you assert that international aid can be problematic for an economy rather than a source of help. Let us say that I am skeptical, that I am unable to see how this can be true. To help me understand this, you construct a little, simplified economic model, in which the increase of foreign economic help generate huge economic difficulties for the country that receives help (for our purposes, we do not need to go into the details of this model). It is then true in this model that if we increase economic help, we deteriorate the economy. If I then ask you whether this model is actually true, you would then say that it is not, and that it does not have to be in order for me to understand how your claim about the downside of foreign help can be true. I then point out that something more has to be assumed. Your model does not have to be true, but it has to be consistent with accepted features of the actual situation. If there is no way in which an economy could possibly satisfy the conditions imposed by your model, the latter would simply be irrelevant to increase my understanding. In other words, a crucial claim of consistency has to be established before any assertion about understanding can be made. And surely you would grant that if your model were inconsistent with accepted information, it would not be of much use to increase my understanding of how your claim about the economy could be true. (Note that even if you were a paraconsistent theorist-who acknowledges that inconsistent theories need not be trivial, in the sense that not everything follows from them-you would still need to provide an account of why, despite its inconsistency with accepted information, the model is still coherent [32].)

What this indicates is the need for a modal notionconsistency-in the characterization of understanding. Can empiricists help themselves to such a notion? As usually formulated, consistency is identified with the existence of an abstract structure (the model of the relevant theory or sentence). But given that an abstract structure is not observable, it is not something an empiricist can believe in. Alternatively, consistency is understood in terms of possibility, as a claim that the relevant theory or sentence is possible, that it can be true. But this means that the empiricist has to assume a primitive notion of modality. It is unclear, however, how to reconcile such primitive notion of modality with the skepticism about modal notions found in constructive empiricism.

It may be argued that constructive empiricists do not have to be skeptical about logical consistency, since this notion is well understood on its own. I think this response makes very good sense. In fact, it is one that I will recommend below. There are, of course, several arguments to the effect that a proper characterization of modal notions requires the introduction of possible worlds or other entities, such as abstract objects, which are not part of the empiricist's ontology [29]. Constructive empiricists should resist these arguments, and offer a modalist account of logical concepts, which recognizes the need for, and the intelligibility of, a primitive logical notion of consistency [33-36]. 
Thus, the example about the economic model illustrates that an account of understanding requires talk of consistency. But it also illustrates where truth steps in. As noted above, it is important that in the economic model your claim about foreign help is true. You will probably say that this is only the notion of truth in a model; what does that have to do with truth?

This is, of course, an important question, and here there seems to be a tension in the use of these two notions in constructive empiricism. In some contexts, perhaps dialectical ones involving debates with the scientific realist, truth is taken as a perfect correspondence between all components of a model of a theory and the world [19,20]; in other contexts, truth is taken in a minimalist sense [27,28]. Truth in a structure becomes crucial in contexts in which one wishes to claim that empirical adequacy is truth about the observable domain. For empirical adequacy is a model-theoretic notion in van Fraassen's hands [19, p.64], and for it to be identified with truth about observables, truth has to be thought of in model-theoretic terms as well. After all, if truth is not conceived in this way, it cannot be a property of a model, as is the case with empirical adequacy. Truth simpliciter is not a norm of science for the constructive empiricist; but this is the notion of truth adopted by scientific realists, and van Fraassen uses it when discussing with them, in order to avoid begging the question against them. The use of a minimalist notion of truth has emerged later in constructive empiricism. The notion is employed when we are talking about our language in use, in contrast with a language that requires interpretation; in the latter case, the notion of truth in an interpretation is adopted [28]. But since the minimalist notion of truth is ontologically committing, this is not a notion that the empiricist can adopt without worry. (Given the following instantiation of the disquotational schema: "There are electrons' is true iff there are electrons", the empiricist cannot claim that the sentence 'There are electrons' is true without being ontologically committed to the existence of electrons. That is why van Fraassen introduces a norm different from truth in the interpretation of science, namely, empirical adequacy.)

Suppose then that these different uses of truth are kept apart and are only explored in appropriate contexts. What is the problem? The problem is that in the context of the modal interpretation, both truth and truth in a structure are used. On the one hand, since the talk of dynamic and value states is not part of our language in use, in the sense that it is not part of our daily vocabulary, it requires an interpretation; and the truth-conditions given to state and value attributions are expressed in terms of a model (the one characterizing the modal interpretation). However, van Fraassen also talks about the truth of quantum me- chanics plus the modal interpretation. On his view, it is in this global setting that we should consider how the world can be if quantum mechanics is true. But in this setting, he is talking about truth simpliciter, for he is considering the world rather than a model of it. We no longer have truth in a structure.

It might be argued that these are two different contexts: one spells out the truth-conditions for state and value attributions (and the empiricist uses truth in a structure); the other determines the truth-conditions of quantum mechanics and the modal interpretation as a whole (and the empiricist uses truth simpliciter). But it is not at all obvious that we have different contexts here. The modal interpretation is, of course, parasitic on quantum mechanics: it is an interpretation of that theory after all. If we were to assert its truth, we would have to assert the truth of quantum mechanics as well. (What I mean here is that, as an interpretation of quantum mechanics, and not as a rival theory, the modal interpretation has to be consistent with quantum theory.) In other words, in the same context-the one determined by quantum mechanicsthe empiricist uses two different notions of truth, and of course the two notions are not equivalent. The problem with this is that truth in a structure is compatible with falsity of the notions under consideration, whereas truth simpliciter is not. (Throughout this discussion I assume with the empiricist that the underlying logic is classical.) So even if the truth-conditions for state-attributions are satisfied, the resulting propositions may actually be false. For the truth-conditions were specified in terms of truth in a structure. For example, suppose that it is true in a model that all frogs are purple provided that they live in a forest. Now, even if it is in fact true that frogs live in forests, it does not follow that frogs are purple. From a semantic viewpoint, this indicates the limitation of the proposed account.

What if the notion of truth in a structure is abandoned, and only truth simpliciter is used to characterize the truthconditions of state attributions? If this is done, no longer will the empiricist be able to claim that the truth of state attributions is not ontologically problematic, since it was only established with respect to a model. (After all, the truth of a state attribution in a given model does not establish its truth.) As a result, the introduction of truthconditions for state attributions becomes ontologically problematic for the empiricist.

But perhaps the empiricist would adopt a different approach. The idea is not to introduce truth in a structure as a way of alleviating ontological commitments to unobservable objects. Truth-conditions are not formulated in terms of a model, but they are expressed as truth simpliciter. The avoidance of ontological commitment arises from the fact that the truth of state attributions is never 
asserted. Rather the empiricist considers quantum mechanics and the modal interpretation as a whole package (that is, quantum mechanics plus the interpretative additions provided by the modal interpretation). In this case, the empiricist will not assert the truth of the resulting theory and interpretation-only the empirical adequacy of the compound matters. And given that empirical adequacy is weaker than truth, and does not depend on the existence of any unobservable entity, it brings no problem for the empiricist.

What this proposal amounts to is the denial that interpretations of quantum mechanics could be evaluated independently of the theory itself. In other words, we should consider quantum mechanics together with each of its interpretations, and evaluate the whole package in terms of its empirical adequacy. Since every interpretation is empirically superfluous (otherwise we would generate a rival theory), the empirical adequacy of any interpretation plus quantum mechanics is guaranteed, provided that the latter is empirically adequate. As a result, as noted above, empirical adequacy is not an informative criterion for the evaluation of interpretations of quantum mechanics. It is not an adequate norm for the interpretation of theories, since every interpretation, in virtue of being an interpretation rather than a rival theory, satisfies it.

After carefully, distinguishing the theory from its interpretations, the empiricist now seems to insist that we should evaluate both as a package. If we are to avoid ontological commitment to unobservable entities, we should only assert the empirical adequacy of the resulting compound. But why should one resist the temptation of asserting the truth of the latter? Except for avoiding the inflationary metaphysics associated with the interpretation, there seems to be no reason why one should not make that move. This means that only empiricists would be persuaded by this suggestion. Is there any reason that constructive empiricists can offer to those who do not share their view, why they should avoid making any claim beyond the empirical adequacy of the compound theory plus interpretation?

Note also that by considering quantum mechanics plus its interpretation as a package only, the constructive empiricist is in no position to assess the truth of such interpretations independently of the theory. This makes it difficult to evaluate critically rival interpretations of quantum mechanics, since any such assessment presupposes that one can demarcate the interpretation from the formalism of the theory, which clearly can be done.

We have now reached the point where the two themes of this section-truth and modality in the modal interpretation-meet. As we saw, truth was introduced in the modal interpretation to separate state-attributing propositions from value-attributing ones. But, given the empiricist constraints of van Fraassen's view, truth determines a norm that is too strong to be applicable to state-attributions. Modality has also been introduced, but here the account seems to be too weak to provide the required philosophical warrant for them. The question arises naturally: Is there an empiricist account that provides both a weaker notion of truth and a stronger account of modality? If so, is this account also appropriate to the particularities of the quantum domain? In order to answer these questions, da Costa and French's partial structures approach provides a useful tool.

\section{Partial Structures and Quasi-Truth}

The partial structures approach (as first presented in [37, 38], and then extended in [21-23]) relies on three main notions: partial relation, partial structure, and quasitruth. (Further developments and applications of this approach can also be found in [24,39-45].) One of the main motivations for introducing this proposal comes from the need for supplying a formal framework in which the openness and incompleteness of information dealt with in scientific practice can be accommodated in a unified way [23]. Two proposals are central to this task. First, the usual concept of structure is extended to model the partialness of information we have about a certain domain, thus leading to the concept of a partial structure. Second, the Tarskian characterization of the concept of truth for such partial contexts is advanced, and the corresponding concept of quasi-truth is formulated.

To characterize a partial structure, one need, first, to formulate an appropriate notion of partial relation. When investigating a certain domain of knowledge $\Delta$ (which is a field of the empirical sciences, such as particle physics), we formulate a conceptual framework that helps us systematize and organize the information we obtain about $\Delta$. This domain is tentatively represented by a set $D$ of objects, and is studied by the examination of the relations holding among $D$ 's elements. However, given a certain relation $R$ defined over $D$, we often do not know whether all the objects of $D$ (or $n$-tuples thereof) are related by $R$. This is part and parcel of the incompleteness of our information about $\Delta$, and is formally accommodated by the concept of partial relation. More formally, let $D$ be a non-empty set; an $n$-place partial relation $R$ over $D$ is a triple $\left\langle R_{1}, R_{2}, R_{3}\right\rangle$, where $R_{1}, R_{2}$, and $R_{3}$ are mutually disjoint sets, with $R_{1} \cup R_{2} \cup R_{3}=D^{n}$, and such that: $R_{1}$ is the set of $n$-tuples that (we know that) belong to $R, R_{2}$ is the set of $n$-tuples that (we know that) do not belong to $R$, and $R_{3}$ is the set of $n$-tuples for which we do not know 
whether they belong or not to $R$. (Note that if $R_{3}$ is empty, $R$ is a usual $n$-place relation that can be identified with $R_{1}$.)

In order to represent the information about the domain under consideration, we need a notion of structure. The following characterization, formulated in terms of partial relations and based on the standard concept of structure, is meant to provide a notion that is broad enough to accommodate the partiality usually found in scientific practice. Partial relations do the main work, of course. A partial structure $S$ is an ordered pair $\left\langle D, R_{i}\right\rangle_{i \in I}$, where $D$ is a non-empty set and $\left(R_{i}\right)_{i \in I}$ is a family of partial relations defined over $D$. (The partiality at stake here is due to the incompleteness of our knowledge about the domain under investigation. Given additional information, a partial relation may become total. Hence, the partiality modeled by the partial structures approach is not understood as an ontological partiality in the world-an aspect about which an empiricist will be glad to remain agnostic. In other words, what is at issue is an epistemic, not an ontological partiality.)

In order to systematize our knowledge of $\Delta$ (say, again, particle physics), the domain $D$ of the partial structure $S$ is typically constituted by two components: (1) observable objects (in the physics of particles, configurations in a Wilson chamber, spectral lines etc.), whose set is denoted by $D_{1}$; and (2) unobservable objects (quarks, for example), whose set is denoted by $D_{2}$. It is understood that $D_{1} \cap D_{2}=\emptyset$, and we require that $D=D_{1} \cup D_{2}$. In this way, the modeling of $\Delta$ involves new partial relations $R_{j}, j \in J$ (defined over $D_{2}$ ), some of which may help to extend the relations $R_{i}, i \in I$ (defined over $D_{1}$ ). As a result, if we want to be explicit, a partial structure $S$ has the following form: $\left\langle D_{1}, D_{2}, R_{i}, R_{j}\right\rangle_{i \in I, j \in J}$. But it is usually easier to refer to it simply as $\left\langle D, R_{k}\right\rangle_{k \in K}$ [22,43].

Two of the three basic notions of the partial structures approach are now defined. To formulate the last, and crucial one-quasi-truth-an auxiliary notion is required. The idea is to use, in the characterization of quasi-truth, the resources offered by Tarski's definition of truth. But since it is only defined for full structures, we have to introduce an intermediary notion of structure to connect full to partial structures. This is the first role of those structures that extend a partial structure $A$ into a full, total structure (which are called A-normal structures). Their second role is purely model-theoretic, namely to put forward an interpretation of a given language and, in terms of it, to characterize basic semantic notions. The question then is: how should $A$-normal structures be defined? Here is an answer. Let $A=\left\langle D, R_{i}\right\rangle_{i \in I}$ be a partial structure. We say that the structure $B=\left\langle D^{\prime}, R_{i}^{\prime}\right\rangle_{i \in I}$ is an $A$-normal structure if (1) $D=D^{\prime}$, (2) every constant of the language in question is interpreted by the same object both in $A$ and in $B$, and (3) $R_{i}^{\prime}$ extends the corresponding relation $R_{i}$ (in the sense that each $R_{i}^{\prime}$, supposed of arity $n$, is defined for all $n$-tuples of elements of $D^{\prime}$ ). Although each $R_{i}^{\prime}$ is defined for all $n$-tuples over $D^{\prime}$, it holds for some of them (the $R_{i 1}^{\prime}$-component of $R_{i}^{\prime}$ ), and it does not hold for others (the $R_{i 2}^{\prime}$-component).

As a result, given a partial structure $A$, there are several $A$-normal structures. Suppose that, for a given $n$-place partial relation $R_{i}$, we do not know whether $R_{i} a_{1} \ldots a_{n}$ holds or not. One way of extending $R_{i}$ into a full $R_{i}^{\prime}$ relation is to look for information to establish that it does hold, another way is to look for the contrary information. Both are prima facie possible ways of extending the partiality of $R_{i}$. But the same indeterminacy may be found with other objects of the domain, distinct from $a_{1}, \ldots, a_{n}$ (for instance, does $R_{i} b_{1} \ldots b_{n}$ hold?), and with other relations distinct from $R_{i}$ (for example, is $R_{j} b_{1} \ldots b_{n}$ the case, with $j \neq i$ ?). In this sense, there are too many possible extensions of the partial relations that constitute $A$. Thus, we need to provide constraints to restrict the acceptable extensions of $A$.

In order to do that, we need first to formulate a further auxiliary notion [37]. A pragmatic structure is a partial structure to which a third component has been added: a set of accepted sentences $P$, which represents the accepted information about the structure's domain. (Depending on the interpretation of science that is adopted, different kinds of sentences are to be introduced in $P$ : realists will typically include laws and theories, whereas empiricists will add mainly certain empirical regularities and observational statements about the domain in question.) A pragmatic structure is then a triple $A=\left\langle D, R_{i}, P\right\rangle_{i \in I}$, where $D$ is a non-empty set, $\left(R_{i}\right)_{i \in I}$ is a family of partial relations defined over $D$, and $P$ is a set of accepted sentences. The idea is that $P$ introduces constraints on the ways that a partial structure can be extended.

The conditions for the existence of $A$-normal structures can now be spelled out [37]. Let $A=\left\langle D, R_{i}, P\right\rangle_{i \in I}$, be a pragmatic structure. For each partial relation $R_{i}$, we construct a set $M_{i}$ of atomic sentences and negations of atomic sentences, such that the former correspond to the $n$-tuples that satisfy $R_{i}$, and the latter to those $n$-tuples that do not satisfy $R_{i}$. Let $M$ be $\bigcup_{i \in I} M_{i}$. Therefore, a pragmatic structure $A$ admits an $A$-normal structure iff the set $M \cup P$ is consistent. (Consistency here is taken as a primitive, logical notion; see [35, 36].)

Assuming that such conditions are met, we can now formulate the concept of quasi-truth. A sentence $\alpha$ is quasi-true in $A$ according to $B$ if (1) $A=\left\langle D, R_{i}, P\right\rangle_{i \in I}$ is a pragmatic structure, (2) $B=\left\langle D^{\prime}, R_{i}^{\prime}\right\rangle_{i \in I}$ is an $A$-normal structure, and (3) $\alpha$ is true in $B$ (in the Tarskian sense). If $\alpha$ is not quasi-true in $A$ according to $B$, we say that $\alpha$ is quasi-false (in $A$ according to $B$ ). Moreover, we say that 
a sentence $\alpha$ is quasi-true if there is a pragmatic structure $A$ and a corresponding $A$-normal structure $B$ such that $\alpha$ is true in $B$ (according to Tarski's account). Otherwise, $\alpha$ is quasi-false.

The idea, intuitively speaking, is that a quasi-true sentence $\alpha$ does not describe the whole domain to which it refers, but only an aspect of it-the one modeled by the relevant partial structure $A$. After all, there are several different ways in which $A$ can be extended to a full structure, and in some of these extensions $\alpha$ may not be true. As a result, the notion of quasi-truth is strictly weaker than truth: although every true sentence is (trivially) quasi-true, a quasi-true sentence is not necessarily true (since it may be false in certain extensions of $A$ ).

To illustrate the use of this notion, let us consider an example. As is well known, Newtonian mechanics is appropriate to explain the behavior of bodies under certain conditions, such as bodies that, roughly speaking, have low velocity in comparison with that of light, are not subject to strong gravitational fields etc. With the formulation of special relativity, we know that if these conditions are not satisfied, Newtonian mechanics is false. In this sense, these conditions specify a family of partial relations, which delimit the context in which the theory holds. Although Newtonian mechanics is not true (and we know under what conditions it is false), it is quasi-true; that is, it is true in a given context, determined by a pragmatic structure and a corresponding $A$-normal one [39].

Having discussed the formulation of partial structures and quasi-truth, we can now consider an application of this conceptual framework to the modal interpretation of quantum mechanics.

\section{Modality, Quasi-Truth and the Modal-Structural Interpretation of Quantum Mechanics}

My suggestion here is that the framework sketched above in terms of partial structures and quasi-truth provides at least a partial answer to the two questions raised at the end of section 3, namely: Is there an empiricist account that provides both a weaker notion of truth and a stronger account of modality? And is this account also appropriate to the particularities of the quantum domain? The idea is that, instead of determining the truth of state-attributions, the empiricist should change the norm of this type of theoretical discourse to quasi-truth. In this way, the commitment to unobservables can be avoided, simply because quasi-truth is weaker than truth. As we saw, in claiming that a sentence $\alpha$ is quasi-true, we are not committed to the claim that it captures, in full detail, all the structure of the domain in question: the relations defined over the domain $D_{2}$ (which concern unobservable aspects of the domain under study) are left open. The only components that are fixed are those that refer to the observable aspects of the domain (which are found, roughly speaking, in $D_{1}$ ). So, from the fact that a theory $T$ (about particle physics) is quasi-true, we cannot conclude that the unobservable aspects of the domain under study are fully described. Note that empirical adequacy has the same feature: if $T$ is empirically adequate, it does not follow that it is true, i.e. that it describes, in full detail, all aspects of the world; the only aspects that are properly accommodated are the observable ones. But since quasi-truth is always relative to a given partial structure (which incorporates both an observable part, $D_{1}$, and an unobservable one, $D_{2}$ ), it readily generalizes van Fraassen's notion of empirical adequacy: roughly speaking, a theory is empirically adequate if it is quasi-true in a partial structure that describes the empirical phenomena. In this sense, quasi-truth is appropriate for an empiricist view (for details, see [24]).

Now, to say that a state-attributing proposition $[m, E]$ is quasi-true-in a partial structure $A$, which represents partial information about the state of the system-is to say that there is an $A$-normal structure $B$ in which $[m, E]$ is true. Note that $B$ provides one possible way of extending the partial information about the state; there may well be different ways of extending it. But this suggests that the concept of quasi-truth already introduces a notion of modality. In fact, this point can be explicitly made as follows (see also [43]): (a) $\square P$ is quasi-true (in a partial structure $A$ ) iff for all $A$-normal structures, $P$ is true. (b) $\diamond P$ is quasi-true (in a partial structure $A$ ) iff there is an $A$-normal structure in which $P$ is true. (The notion of relative possibility can be introduced without difficulty.) Now, as defined, these modal operators are clearly about the structures under consideration, namely those employed to represent the states of a given physical system. In order to use these operators, there is no need to go beyond the language used to describe such states. There is no need for the introduction of possible worlds, since the modal operators are explicitly formulated in terms of quasi-truth. Moreover, only a primitive notion of logical consistency is assumed in the background, as part of the partial structures approach. But there are independent reasons to favor this form of modalism, given the need for such a primitive notion in order to make sense of logical consequence and logical constants [33-36]. In the end, all we have to do is explore the representation possibilities of the relevant structures. In this sense, the proposal advanced here accommodates straightforwardly the constructive empiricist's point that modal talk is concerned with the structures we use to represent the phenomena.

But the question of the adequacy of the modal operators formulated via quasi-truth immediately arises. Can we 
obtain results about such operators analogous to those established in van Fraassen's interpretation? In particular, can we establish that:

\section{$[m, E]$ is quasi-true iff $\square\langle m, E\rangle$ is quasi-true?}

This is, of course, the crucial modal result (in terms of quasi-truth) of the modal interpretation, highlighting a consequence by which, in van Fraassen's words, the interpretation "merits at once the epithet "modal" [13, p.314]. The result is not difficult to establish. First, let us assume that $\square\langle m, E\rangle$ is quasi-true. Thus, for all $A$-normal structures $B,\langle m, E\rangle$ is true; in other words, in each $B$ the observable $m$ actually has a value in $E$ (in symbols: $\lambda(w)(m) \subseteq E$, where $\lambda(w)$, the value state, is taken as a map from observables into non-empty Borel sets). Now, since this holds for each $B$, there is an $A$-normal structure $B^{\prime}$ in which the state is such that the probability that the observable $m$ has a value in $E$ is one (in symbols: $\left.P_{x}^{m}(E)=1\right)$. Hence, $[m, E]$ is true in $B^{\prime}$, and therefore it is quasi-true. Second, let us assume that $\square\langle m, E\rangle$ is not quasi-true. In this case, there is no $A$-normal structure $B$ in which $\langle m, E\rangle$ is true; that is, in no $B$ does the observable $m$ have a value in $E$. Thus, there is no $B$ in which the state is such that the probability that $m$ has a value in $E$ is one. Therefore, $[m, E]$ is not quasi-true. This concludes the proof.

It is thus established that state-attributing propositions are equivalent to the necessitation of value-attributions. This is expected given the definition of state-attributions: they determine a strong, modal claim about the state of a physical system. The result helps us to see the explicit role that is played by modal notions in the interpretation of quantum mechanics since it provides a way of representing the possibilities open to the evolution of a physical system and the interrelationships between the modal operators that are formulated.

It is worth noting that, by moving to the partial structures approach, we can also represent an important feature of van Fraassen's modal interpretation. As we saw, van Fraassen's rejection of von Neumann's interpretation rule allowed the introduction of unsharp values to observables. Now, such unsharp values may be assigned to an observable $m$ by a value state $\lambda$. As van Fraassen indicates, one way of spelling this out " is to say that $\lambda$ is a partial function assigning values to some observables but not to others" [13, p.307]. Although van Fraassen does not adopt this alternative, it is clear that, within the partial structures approach, this move can be represented straightforwardly. If we view a value state as a partial function (which is a particular kind of partial relation), the unsharp values are those for which $\lambda$ is not defined (given our current knowledge about the state of the physical system under consideration). It then becomes clear why we need the necessitation of value-attributions to make them equivalent to state attributions: intuitively speaking, all possibilities have to be covered.

Thus, by moving to partial structures and quasi-truth, the two problems faced by van Fraassen's modal interpretation can be overcome: we do not have to talk about the truth of state-attributing propositions (and can remain empiricist about them), and there is a straightforward way of introducing modality into the modal interpretation (via the notion of quasi-truth), which satisfies strictly empiricist constraints.

\section{Conclusion}

Although far more could be said, I hope to have indicated that the present framework provides a plausible setting for the empiricist to investigate the quantum domain. Given the role played by structures and modal operators in the approach, it seems adequate to call it a modal-structural interpretation. If developed further, it provides an interesting way of examining, in modal and empiricist terms, some of the peculiarities of the foundations of quantum mechanics.

\section{Acknowledgements}

My thanks go to Newton da Costa, Steven French, Décio Krause, and Bas van Fraassen for helpful discussions. Thanks are also due to two reviewers, whose identity has now been revealed to me, Eliahu Cohen and Danko Georgiev, for their many insightful suggestions and advice.

\section{References}

[1] von Neumann J. Mathematical Foundations of Quantum Mechanics. Beyer RT (translator), Princeton: Princeton University Press, 1955.

[2] Birkhoff G, von Neumann J. The logic of quantum mechanics. Annals of Mathematics 1936; 37 (4): 823-843. http://www.jstor.org/stable/ 1968621

[3] Putnam H. Is logic empirical? In: Proceedings of the Boston Colloquium for the Philosophy of Science, 1966-1968. Boston Studies in the Philosophy of Science, vol.5, Cohen RS, Wartofsky MW (editors), Dordrecht: D. Reidel, 1968, pp.216-241.

[4] Bub J. The Interpretation of Quantum Mechanics. Dordrecht: D. Reidel, 1974. 
[5] Rédei M. Quantum Logic in Algebraic Approach. Dordrecht: Kluwer Academic Publishers, 1998.

[6] Manin YI. A Course in Mathematical Logic for Mathematicians, 2nd edition. Graduate Texts in Mathematics, vol.53, New York: Springer, 2010.

[7] da Costa NCA, Krause D. Schrödinger logics. Studia Logica 1994; 53 (4): 533-550. http://dx. doi org/10.1007/BF01057649

[8] da Costa NCA, Krause D. An intensional Schrödinger logic. Notre Dame Journal of Formal Logic 1997; 38 (2): 179-194.

[9] French S, Krause D. Vague identity and quantum non-individuality. Analysis 1995; 55 (1): 2026. http://dx.doi.org/10.1093/analys/55. 1.20

[10] Krause D, French S. A formal framework for quantum non-individuality. Synthese 1995; 102 (1): 195-214. http://dx.doi.org/10.1007/ BF01063905

[11] French S, Krause D. Identity in Physics: A Historical, Philosophical, and Formal Analysis. Oxford: Clarendon Press, 2006.

[12] Redhead M. Incompleteness, Nonlocality, and Realism: A Prolegomenon to the Philosophy of Quantum Mechanics. Oxford: Oxford University Press, 1987.

[13] van Fraassen BC. Quantum Mechanics: An Empiricist View. Oxford: Clarendon Press, 1991.

[14] Dummett M. Is logic empirical? In: Contemporary British Philosophy: Fourth Series. Lewis HD (editor), London: Allen and Unwin, 1976, pp.45-68. Reprinted in Dummett M, Truth and Other Enigmas, Cambridge, MA: Harvard University Press, 1978, pp.269-289.

[15] Dieks D, Vermaas PE. The Modal Interpretation of Quantum Mechanics. The Western Ontario Series in Philosophy of Science, Dordrecht: Kluwer, 1998.

[16] van Fraassen BC. A formal approach to the philosophy of science. In: Paradigms and Paradoxes: The Philosophical Challenge of the Quantum Domain. University of Pittsburgh Series in the Philosophy of Science, Colodny RG (editor), Pittsburgh: University of Pittsburgh Press, 1972, pp.303-366.

[17] van Fraassen BC. Semantic analysis of quantum logic. In: Contemporary Research in the Foundations and Philosophy of Quantum Theory, vol.2. The
University of Western Ontario Series in Philosophy of Science, Hooker CA (editor), Springer Netherlands, 1973, pp.80-113.http://dx.doi.org/10. 1007/978-94-010-2534-8_3

[18] van Fraassen BC. The Einstein-Podolsky-Rosen paradox. Synthese 1974; 29 (1-4): 291-309. http: //dx.doi.org/10.1007/BFQ0484962

[19] van Fraassen BC. The Scientific Image. Oxford: Clarendon Press, 1980.

[20] van Fraassen BC. Laws and Symmetry. Oxford: Clarendon Press, 1989.

[21] da Costa NCA, French S. Pragmatic truth and the logic of induction. The British Journal for the Philosophy of Science 1989; 40 (3): 333-356. http: //dx.doi.org/10.1093/bjps/40.3.333

[22] da Costa NCA, French S. The model-theoretic approach in the philosophy of science. Philosophy of Science 1990; 57 (2): 248-265. http://www, jstor.org/stable/187834

[23] da Costa NCA, French S. Science and Partial Truth: A Unitary Approach to Models and Scientific Reasoning. Oxford Studies in Philosophy of Science, New York: Oxford University Press, 2003.

[24] Bueno O. Empirical adequacy: A partial structures approach. Studies in History and Philosophy of Science A 1997; 28 (4): 585-610. http://dx.doi. org/10.1016/S0039-3681(97)00012-5

[25] van Fraassen BC. From a view of science to a new empiricism. In: Images of Empiricism: Essays on Science and Stances, with a Reply from Bas van Fraassen. Mind Association Occasional Series, Monton B (editor), Oxford: Clarendon Press, 2007, pp.337-383.

[26] van Fraassen BC. The Empirical Stance. The Terry Lectures Series, New Haven: Yale University Press, 2002.

[27] van Fraassen BC. Structure and perspective: philosophical perplexity and paradox. In: Logic and Scientific Methods: Volume One of the Tenth International Congress of Logic, Methodology and Philosophy of Science, Florence, August 1995, vol.259. Synthese Library, Dalla Chiara ML, Doets K, Mundici D, Benthem J (editors), Springer Netherlands, 1997, pp.511-530. http://dx.doi.org/ 10.1007/978-94-017-0487-8_29 
[28] van Fraassen BC. Elgin on Lewis's Putnam's paradox. Journal of Philosophy 1997; 94 (2): 85-93. http://www . jstor.org/stable/2940777

[29] Lewis DK. On the Plurality of Worlds. Oxford: Blackwell, 1986.

[30] Monton B, van Fraassen BC. Constructive empiricism and modal nominalism. British Journal for the Philosophy of Science 2003; 54 (3): 405-422. http: //dx.doi.org/10.1093/bjps/54.3.405

[31] van Fraassen BC. The only necessity is verbal necessity. Journal of Philosophy 1977; 74 (2): 71-85. http://www.jstor.org/stable/2025572

[32] Bueno O, da Costa NCA. Quasi-truth, paraconsistency, and the foundations of science. Synthese 2007; 154 (3): 383-399. http://dx.doi.org/10. $1007 / \mathrm{s} 11229-006-9125-\mathrm{x}$

[33] Bueno O, Shalkowski SA. A plea for a modal realist epistemology. Acta Analytica 2000; 15 (24): 175-193. http://eprints.whiterose.ac. uk/3354/

[34] Bueno O, Shalkowski SA. Modal realism and modal epistemology: a huge gap. In: Modal Epistemology. Weber E, De Mey T (editors), Brussels: Royal Flemmish Academy of Belgium, 2004, pp.93-106.

[35] Bueno O, Shalkowski SA. Modalism and logical pluralism. Mind 2009; 118 (470): 295-321. http: //dx.doi.org/10.1093/mind/fzp033

[36] Bueno O, Shalkowski SA. Logical constants: a modalist approach. Noûs 2013; 47 (1): 1-24. http://dx.doi.org/10.1111/j.1468-0068. $2012.00865 . \mathrm{x}$

[37] Mikenberg I, da Costa NCA, Chuaqui R. Pragmatic truth and approximation to truth. Journal of Symbolic Logic 1986; 51 (1): 201-221. http: //wwW.jstor.org/stable/2273956
[38] da Costa NCA. Pragmatic probability. Erkenntnis 1986; 25 (2): 141-162. http://dx.doi.org/10. 1007/BFO0167168

[39] da Costa NCA, French S. Towards an acceptable theory of acceptance: Partial structures, inconsistency and correspondence. In: Correspondence, Invariance and Heuristics, Boston Studies in the Philosophy of Science, vol.148, French S, Kamminga H (editors), Springer Netherlands, 1993, pp.137-158. http://dx.doi.org/ 10.1007/978-94-017-1185-2_7

[40] da Costa NCA, French S. A model theoretic approach to 'natural' reasoning. International Studies in the Philosophy of Science 1993; 7 (2): 177-190. http://dx.doi.org/10.1080/ Q2698599308573462

[41] da Costa NCA, French S. Partial structures and the logic of Azande. American Philosophical Quarterly 1995; 32 (4): 325-339. http://www. jstor.org/ stable/20009835

[42] French S. Partiality, pursuit and practice. In: Structures and Norms in Science: Volume Two of the Tenth International Congress of Logic, Methodology and Philosophy of Science, Florence, August 1995. Dalla Chiara ML, Doets K, Mundici D, van Benthem J (editors), Dordrecht: Kluwer Academic Publishers, 1997, pp.35-52.

[43] da Costa NCA, Bueno O, French S. The logic of pragmatic truth. Journal of Philosophical Logic 1998; 27 (6): 603-620. http://dx.doi.org/10. 1023/A\%3A1004304228785

[44] Bueno O. What is structural empiricism? Scientific change in an empiricist setting. Erkenntnis 1999; 50 (1): 55-81.http://dx.doi.org/10.1023/A\% 3A1005434915055

[45] Bueno O, de Souza EG. The concept of quasi-truth. Logique et Analyse 1996; 39 (153-154): 183-199. 